\title{
EDITORIAL
}

\section{The New Dimension of Endocrinology: Nuclear/Intracellular Endocrinology}

\author{
SHIGEAKI KATO
}

Professor of Institute of Molecular and Cellular Biosciences, University of Tokyo, Tokyo 113-0032, Japan

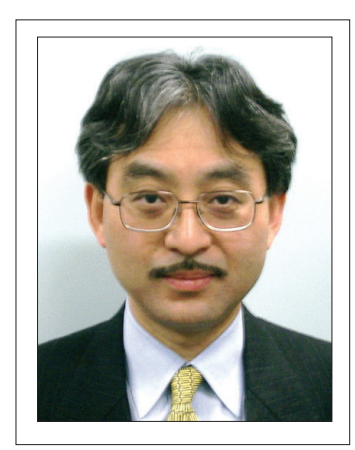

\section{Endocrinology is Developing}

Endocrinology has been fruitfully developed to date by incorporating and merging with related and current biomedical fields. Recent successes in diverse approaches to generate new fields of modern biology, such as structural biology, system biology, and chromatin-landscape survey, is expected to accelerate the development of endocrinology. Though modern devices and techniques have been improved and invented, the central issue of endocrinology is no doubt dependent on the advance of conceptual theories to understand endocrine-related events. In this respect, here I would like to discuss the possible application of epigenome to modern endocrinology.

\section{Canonical Epigenome vs Modern Epigenome}

The definition of "epigenome" seems to be more flexible to cover many aspects related with chromatin reconfiguration. Epigenome does not mean a substance such as a gene, but is a concept of heritable changes without alterations in DNA sequence. Originally and canonically speaking, DNA methylation to discriminate maternal from paternal genetic heritage has been considered as "epigenomic." Sexdependent expression of several genes like IGF-I is well characterized and such sex difference in gene expression has been uncovered owing to the DNA methylation patterns in the gene promoters. Once DNA methylation is induced in somatic cells, the DNA methylation pattern was initially believed to remain unaltered. However, DNA methylation to inactivate chromatin, such as heterochromatinization, is found to be induced by or coupled with tumorigenesis in many tissues including hormone-dependent organs. At present, the precise detection of DNA methylated sites on the genome-wide scale has been hampered by techni- cal difficulties. DNA methylation is now under consideration as DNA methylation patterns may be reversible and changeable under certain physiological and pathological conditions. This idea is supported by the fact that differentiated cells are still potent enough to be converted into iPS cells, presumably coupling with altered DNA methylation pattern.

Beyond canonical epigenomic modification, the current view of the epigenome includes histone modifications. Many types of post-translation modifications have been recorded on the N-terminal ends of histones. The combination of histone modifications is assumed to direct the chromatin state, and this assumption is proposed as a theory designated as the "histone code" (in 1999). Histone acetylation was firstly found in 1995 to be closely related with the state of activated chromatin like euchromatin. Indeed enzymes such as p300/CBP to acetylate histones are known to co-activate the transactivation function of DNA-binding transcription factors, while histone deacetylases (HDACs) serve as transcriptional co-repressors through histone inactivation by histone deacetylation. Similar analyses have been performed to characterize histone modifications and their responsible enzymes, and basically could verify the hypothesis of the "histone code." Among the histone modifications, histone methylation has emerged as the most upstream and critical histone mark directing the chromatic state, but appears unlikely to resemble histone acetlylation. Histone $\mathrm{H} 3$ lysine 4 and 36 methylations (H3K4 and H3K36) activate the chromatin state, while histone inactivation such as heterochromatin is induced by methylations of H3K9 and H3K27. Dependent on the lysine residues on histone N-tails, histone methylation leads in the opposite direction. Besides such well-documented modifications, unknown modifications on histones are still 
being studied. Thus, histone modifications are significant determinants for gene expression on chromosome, and hence they are now recognized as a class of epigenomic modifications.

\section{A New Era of Nuclear/Intracellular Endocrinology}

Intracellular endocrinology has come of age. By success of the field, modern endocrinologists to date are familiar with the idea that intracellular signalings triggered by either endocrine hormones or endocrineunrelated ligands are parts of the global endocrine systems. Intracellular systems are nowadays issues for endocrinology. The paradigm for places where endocrine systems are at work has shifted to cover intracellular systems. This shift was successfully achieved by the advance of experimental approaches in molecular and cellular biology. Likewise, it is quite conceivable from the rapid progress of epigenomic fields that a new field of endocrinology will be launched (or has already been launched), and it might be named "Nuclear Endocrinology." Histone modifying enzymes act as epigenetic regulators and form nuclear complexes in many cases. Such complexes most likely co-regulate the function of distinct classes of nuclear factors through their direct and indirect interactions. The complexes thus appear to mediate the cross-talk between (among) distinct intracellular signalings. Furthermore, our group could provide several representative examples that the complex components are downstream targets for intracellular signalings activated by cellular and cell membrane receptors. Since most of the biological outputs from diverse endocrine systems mediate gene expression, it is evident that downstream signals merge on epigenomic regulators/ complexes. "Nuclear Endocrinology" is thus expected to illuminate such epigenomic aspects linking intracellular signaling pathways to nuclear events. 